\title{
Estudo de Validação da Escala de Autoeficácia para Psicólogos em Contexto Escolar
}

\author{
Solange Ester Koehler ${ }^{1}$ \\ ISPA - Instituto Universitário, Lisboa, Portugal \\ Instituto Federal Farroupilha, Santa Maria-RS, Brasil \\ Lourdes Mata \\ ISPA - Instituto Universitário, Lisboa, Portugal
}

\section{RESUMO}

Considerando a inexistência de instrumentos de autoeficácia ocupacional direcionada aos psicólogos em contexto escolar, este estudo pretende descrever a construção e a validação de uma escala, além de apresentar as qualidades psicométricas da Escala de Autoeficácia para Psicólogos em Contexto Escolar - EAPsi. Utilizou-se como embasamento a Teoria Social Cognitiva, que explica o comportamento humano por meio da proatividade, da autorregulação e da auto-organização. Esta pesquisa contou com a participação de 205 psicólogos atuantes nos Institutos Federais de Educação, Ciência e Tecnologia. Essas instituições compõem a Rede Federal de Educação Profissional, Científica e Tecnológica que está presente em todos os estados do território nacional. Constatou-se que $80 \%$ eram do gênero feminino, com idade média de 35 anos. Os resultados da análise fatorial exploratória indicaram que a estrutura com 22 itens e três dimensões foi a mais ajustada, com alfas de Cronbach indicando boa consistência interna. As dimensões foram: autoeficácia profissional, autoeficácia relacional e autoeficácia na articulação com as famílias. Assim sendo, a validação da EAPsi contribui para preencher uma carência de instrumentos voltados aos profissionais, podendo colaborar com estudos e intervenções na área.

Palavras-chave: psicólogos escolares; autoeficácia; escala; validação.

\section{ABSTRACT - Validation of the School Psychologists Self-Efficacy Scale}

Considering the lack of instruments related to occupational self-efficacy directed toward psychologists in a school context, this study aimed to describe the construction and validation of a scale and to present the psychometric qualities of the Self-efficacy Scale for Psychologists in the School Context - EAPsi. Social Cognitive Theory was used to explain human behavior through proactivity, selfregulation and self-organization. This study had the participation of 205 psychologists working in the Federal Institutes of Education, Science and Technology. These institutions make up the Federal Network of Professional, Scientific and Technological Education that is present in all the states of the country. It was found that $80 \%$ of the participants were female, with an mean age of 35 years. The results of the exploratory factor analysis indicated that the structure with 22 items and 3 dimensions presented the best fit, with Cronbach's alphas indicating good internal consistency. The dimensions were: professional self-efficacy, relational self-efficacy and self-efficacy in articulation with families. Accordingly, the validation of the EAPsi contributes to fill a gap in instruments aimed at professionals and presents the possibility for use in studies and interventions in the area.

Keywords: school psychologists; self-efficacy; scale; validation.

\section{RESUMEN - Estudio de Validación de la Escala de Autoeficacia para Psicólogos en Contexto Escolar}

Considerando la existencia de los instrumentos de autoeficacia ocupacional direccionada a los psicólogos en contexto escolar, lo pretendido estudio busca describir la construcción y la validación de una escala, además de presentar las calidades psicométricas de la Escala de Autoeficacia para Psicólogos en Contexto Escolar - EAPsi. Se utilizó como fundamentación la Teoría Social Cognitiva, que explica el comportamiento humano por medio de la proactividad, de la autorregulación y de la autoorganización. La referida investigación abordó 205 psicólogos actuantes en los Institutos Federales de Educación, Ciencia y Tecnología. Estas instituciones componen la Red Federal de Educación Profesional, Científica y Tecnológica presente en todas las provincias del territorio nacional. Se constató que $80 \%$ de los psicólogos eran del género femenino, con edad media de 35 años. Los resultados del análisis factorial exploratorio indicaron que la estructura con 22 aspectos de análisis y tres dimensiones fue la más ajustada, con alfas de Cronbach indicando buena consistencia interna. Las dimensiones fueron: autoeficacia profesional, autoeficacia relacional y autoeficacia en colaboración con las familias. Siendo así, la validación de la EAPsi contribuye para suplir la carencia de instrumentos orientados a los profesionales, pudiendo colaborar con estudios e intervenciones en el área.

Palabras clave: psicólogos escolares; autoeficacia; escala; validación.

Sabe-se que hoje em dia os psicólogos atuantes em contexto escolar são profissionais muito requeridos por educadores, pela equipe escolar e pelas famílias, levando a grandes desafios e diversidade na sua atuação (Fagan, 
1992; Jimerson, 2016; Jimerson, Skokut, Cardenas, Malone \& Stewart, 2008; Lopes \& Almeida, 2015; Oakland \& Jimerson, 2007). Assim, esta pesquisa procurou desenvolver um instrumento que permita compreender quão confiantes esses psicólogos se sentem no desenvolvimento das diferentes facetas de sua atividade profissional.

Pode-se constatar que a atuação dos psicólogos em contexto escolar vem se afirmando como campo de atuação, pesquisa e produção de conhecimento (Jimerson et al., 2008; Jimerson, Stewart, Skokut, Cardenas \& Malone, 2009). Ademais, a forma como se concebe a atuação dos psicólogos em contexto escolar pode ser muito diferenciada. Determinadas perspectivas concebem o papel do psicólogo de maneira direcionada, atuando sobre casos e indivíduos específicos com enfoque essencialmente na avaliação psicológica e no seu acompanhamento ou encaminhamento. Por outro lado, existem perspectivas que defendem a atuação do psicólogo para além da seleção e classificação de alunos com problemas, demonstrando uma visão mais ampla da Psicologia que a enquadra em um compromisso social mais geral (Bahr et al., 2017; Guzzo, 2015; Helgoth \& Sobansky, 2008; Petroni \& Souza, 2014).

Esta última abordagem, considerada mais abrangente, ou institucional, procura focar em ações preventivas para promover o desenvolvimento integral dos sujeitos, dos grupos e das relações existentes no espaço escolar. Essa forma de atuação considera como dimensões centrais as ações associadas ao acompanhamento psicológico e acompanhamento do processo de ensino e de aprendizagem, à assessoria do trabalho de profissionais e ao conhecimento e intervenção a nível institucional (Bahr et al., 2017; Marinho-Araujo \& Almeida, 2014).

A atuação preventiva da Psicologia em contexto escolar visa a construção de estratégias diversificadas, desenvolvendo ações em conjunto com os professores e demais membros da equipe escolar a fim de promover uma tomada de consciência sobre as funções, papéis e responsabilidades dos diferentes intervenientes que atuam em conjunto. Percebe-se a necessidade de analisar as relações existentes no espaço escolar e como isso influencia o sucesso dos alunos. Acrescido a necessidade de priorizar a valorização das relações interpessoais que são essenciais e que enquadram toda a intervenção do psicólogo. Essa perspectiva é uma alternativa para práticas que entendem os fenômenos educativos com um foco individual, nos problemas e dificuldades do aluno (Marinho-Araujo \& Almeida, 2014).

Tal forma de conceber a intervenção dos psicólogos - mais multifacetada e generalizada em relação ao público-alvo, aos objetivos e estratégias implementadas - tem sido refletida e ilustrada em alguns estudos de caraterização em diferentes contextos e realidades culturais (Almeida \& Peixoto, 2011; Bertollo-Nardi, 2014; Jimerson, 2016). Podem ser citados exemplos dos desafios enfrentados pelo profissional de Psicologia no atual contexto, como o aumento do número de alunos nas escolas; as novas configurações de família; as opções sexuais diferenciadas; o número elevado de transtornos mentais; o processo de inclusão de alunos com necessidades especiais, entre outros.

Assim, partindo dessas ações e conforme surgem novas problemáticas na sociedade, são exigidas novas competências e abordagens desse profissional. Um dos fatores associados à escolha de estratégias e persistência na procura de alternativas são os julgamentos de autoeficácia. Autoeficácia entendida como o quão o indivíduo se percebe competente diante das atividades específicas (Bandura, 1977, 2012). Devido à centralidade desse conceito, nos últimos anos foram desenvolvidos estudos com o intuito de analisar a relação existente entre os julgamentos e a atuação, priorizando o bem-estar e a satisfação de profissionais em diferentes áreas (Consiglio, Borgogni, Tecco \& Schaufeli, 2016; Holzberger, Philipp \& Kunter, 2013; Shoji et al., 2016; Skaalvik \& Skaalvik, 2014).

\section{Autoeficácia na perspetiva da Teoria Social Cognitiva}

A autoeficácia é um construto atribuído ao pesquisador Albert Bandura como parte da Teoria Social Cognitiva (TSC), a qual é continuamente aprimorada por seu idealizador e demais pesquisadores (Bandura, 2008; Pajares \& Olaz, 2008). Essa teoria vem sendo pesquisada em diferentes contextos, levando a um aprofundamento dos constructos e à compreensão da sua abrangência, tais como saúde, educação, organizacional/profissional, esporte, entre outros.

Segundo os pressupostos da TSC, o pensamento humano e a ação humana são considerados produtos de uma inter-relação dinâmica entre várias influências, como pessoais, comportamentais e ambientais (Bandura, 2008). Desse modo, destaca-se o papel ativo atribuído aos indivíduos no processo de aprendizagem e a sua interlocução com o ambiente, que se torna o principal mediador desse processo. Um dos fundamentos da TSC se refere à maneira como as relações com o ambiente são organizadas, considerando o comportamento e o indivíduo. Esses três fatores - ambiente, comportamento e indivíduo - operam de maneira conjunta, ou seja, são determinantes interconectados uns aos outros e, portanto, conceituados como determinismo recíproco (Bandura, 1986; Pajares \& Olaz, 2008).

No que concerne à autoeficácia, Bandura (1977, 1986, 2012) caracteriza como um elemento necessário, que faltava para a explicação dos processos de mudança psicológica. Trata-se de crenças que o indivíduo possui em relação à sua capacidade de organização e execução de ações requeridas para lidar com situações futuras; em outras palavras, as crenças referem-se à forma como o indivíduo avalia sua capacidade de desempenhar 
determinadas ações com sucesso. Dessa maneira, os julgamentos de autoeficácia de uma pessoa podem determinar seu nível de motivação, já que é em função desses julgamentos que o indivíduo tem incentivo para agir e pode antecipar mentalmente suas ações para obter resultados.

A partir das crenças de autoeficácia, torna-se possível compreender o estabelecimento de metas, a quantidade de esforço que o profissional depreende e a perseverança para atingir os objetivos. Contudo, isso não garante o sucesso das ações, mas pode determinar a quantidade de energia empreendida na tarefa (Bandura, 1977, 2012).

Para Bandura e colaboradores (Bandura, 1977, 1997, 2012; Pajares \& Olaz, 2008), a construção dos julgamentos de autoeficácia ocorre por meio de quatro fontes de informações: a experiência direta (de domínio ou de êxito); a experiência vicária (ou indireta); a persuasão social; e os estados físicos e emocionais. A experiência direta se baseia em resultados das próprias experiências, pois o êxito obtido na realização de alguma atividade é avaliado como positivo e derivado do esforço da superação de problemas presentes nas ações realizadas. Assim, aumentam as chances de o indivíduo persistir, expandindo também a crença na sua própria capacidade de executar suas ações. O foco nas experiências diretas considera: (a) as ações anteriores; (b) o esforço necessário para obter sucesso; (c) a abrangência e as dificuldades da própria tarefa a ser desenvolvida; (d) a quantidade de apoio externo existente; (e) as demandas da situação na qual a tarefa será realizada também aponta que essa fonte teórica é considerada a de maior influência, uma vez que se baseia em uma experiência de sucesso autêntica. $\mathrm{O}$ autor destaca ainda que o sucesso em dadas ações tende a aumentar as apreciações sobre a eficácia pessoal, enquanto o fracasso repetido tende a diminuir a crença de autoeficácia, especialmente quando ocorre cedo no curso dos eventos e quando não há circunstâncias externas adversas.

A experiência vicária é considerada outra via para auxiliar na construção das crenças de autoeficácia, uma vez que abrange o conjunto de experiências nas quais o sujeito não é participante ativo da tarefa-alvo, mas o observador. Essas experiências se relacionam com os modelos sociais analisados e seguidos, considerando que, ao observar outras pessoas realizarem determinada tarefa, o sujeito pode perceber-se também capaz de fazê-lo (Bandura, 2008). Pajares e Olaz (2008), as pessoas buscam modelos que possuem as competências almejadas por elas; essa similaridade funciona positivamente e influencia a autoeficácia.

A persuasão social refere-se a outro tipo de influências, que funcionam pelo convencimento de uma pessoa sobre suas capacidades. Normalmente, busca-se persuadir verbalmente o sujeito para aumentar o esforço e a superação dos desafios presentes na atividade. A persuasão verbal ocorre por meio dos feedbacks sobre o desempenho na tarefa em questão, dependendo da credibilidade, do conhecimento e da experiência que possui a fonte persuasiva na atividade em questão (Bandura, 2012).

Por fim, os estados fisiológicos e emocionais se referem aos indicadores (ou seja, aos sinais físicos) usados como um meio de avaliar a capacidade ou a disfunção em uma atividade, como o humor e a ansiedade (Bandura, 1986, 1995, 1997, 2012). Pode-se citar também manifestações como a fadiga, o estresse, a ansiedade, a tensão, a dor e os estados de humor, por exemplo, o que pode afetar no julgamento que as pessoas fazem sobre a própria capacidade de realizar determinada tarefa. Bandura (2001) alerta ainda para a forma como lidamos com as manifestações fisiológicas que influenciam a autoeficácia. As pessoas podem avaliar o grau de confiança pelo seu estado fisiológico - enquanto se pensa em uma ação para aumentar as crenças de autoeficácia, é preciso promover o bem-estar emocional e reduzir os estados emocionais negativos.

As diferentes fontes de autoeficácia raramente operam de maneira independente, já que as pessoas não apenas vivenciam o resultado de seus esforços, mas também observam outros indivíduos em situações similares. Além disso, as avaliações sociais sobre a adequação da performance são realizadas periodicamente (Azzi \& Vieira, 2014). Para Pajares e Olaz (2008) as crenças de autoeficácia ajudam o indivíduo a decidir sobre a seleção de tarefas, o que deve contribuir para que ele se sinta mais competente e confiante para executá-las. Conforme o autor (Bandura, 1977, 1997) o sujeito que possui uma autoeficácia elevada em determinada tarefa não apresenta necessariamente o mesmo resultado em uma tarefa distinta. Ademais, a autoeficácia não apresenta uma estabilidade temporal, por vezes o sucesso ou o fracasso podem alterar a percepção do indivíduo em relação às crenças em uma tarefa específica.

\section{Autoeficácia em contexto educativo}

A autoeficácia se relaciona com a capacidade do profissional em executar determinadas ações, que podem gerar e unificar pensamentos, sentimentos e habilidades requeridas para sua execução. Sabe-se que o construto de autoeficácia é específico e relativo a diversos domínios (Ambiel \& Noronha, 2012; Bandura, 2008; Moreira, Ambiel \& Nunes, 2018; Nunes, 2008). Em contexto educativo são muitos os estudos desenvolvidos com alunos e professores, porém, especificamente no Brasil ainda há poucos. É importante salientar que autoeficácia em contexto escolar (Guerreiro-Casanova, 2015; Polydoro \& Guerreiro-Casanova, 2010) é o conjunto de crenças e de expectativas acerca das capacidades pessoais em realizar atividades e trocas, concretizar objetivos e alcançar resultados especificamente na realização escolar.

Tais trabalhos têm evidenciado a importância da autoeficácia para a compreensão de muitos aspetos, como 
os processos motivacionais, o desempenho, a qualidade da ação educativa e o bem-estar (Fonsêca, Couto, Silva, Coelho \& Carvalho, 2018; Höltge, Ehm, Hartmann \& Hasselhorn, 2017; Kim \& Seo, 2018; Poulou, Reddy \& Dudek, 2018; Talsma, Schüz, Schwarzer \& Norris, 2018; Zee \& Koomen, 2016). Ainda são poucas as pesquisas direcionadas ao estudo da autoeficácia de outros profissionais que atuam em contexto escolar, e a escassez de trabalhos pode estar relacionada com aspectos muito específicos da atuação dos psicólogos, como o aconselhamento de carreira e a consultoria (Guiney, Harris, Zusho \& Cancelli, 2014; Watt et al., 2019).

Assim, compreendendo a autoeficácia como uma ferramenta específica para determinadas atividades e contextos, e considerando que não há instrumentos para os profissionais da Psicologia que atuam em contexto escolar em diferentes vertentes de atuação, optou-se por elaborar uma escala para avaliar a autoeficácia de psicólogos em contexto escolar, contemplando não só suas diferentes tarefas, mas também os contextos de atuação.

\section{Método}

\section{Participantes}

Participaram dessa pesquisa 205 psicólogos escolares, servidores dos 38 Institutos Federais (45\% do total de profissionais na rede federal de ensino), representando todos os estados do Brasil. Os IFs atendem prioritariamente alunos do ensino médio, com ênfase no ensino técnico, além de discentes de cursos tecnólogos, bacharelados, graduações e pós-graduação lato e stricto sensu. Essas instituições tiveram sua origem no Brasil em 1909, com a criação das Escolas de Aprendizes e Artífices destinadas ao ensino profissional primário gratuito para os menos favorecidos economicamente (Frigotto, 2018). A partir de 2008, as instituições foram reordenadas e multiplicadas e, atualmente, os 38 IFs estão presentes em todos os estados, com mais de 644 unidades de ensino, compreendendo espaços pluricurriculares e multicampi especializados na oferta da educação profissional e tecnológica em níveis da educação básica, profissional e superior (Pacheco, 2011), totalizando 90\% da rede federal de educação profissional, científica e tecnológica. Os psicólogos inseridos na equipe multi ou interdisciplinar (Bisinoto \& Marinho-Araujo, 2009; Cavalcante, Siqueira \& Kuniyoshi, 2014; Feitosa, 2017) dos IFs no Brasil respondem de maneira diversificada às oportunidades e às demandas de atuação em contextos educativos. A idade dos participantes variou entre 24 e 60 anos, com idade média de $35,44(D P=6,79)$. As mulheres representaram $80 \%$ da amostra, sendo a maioria $(54,4 \%)$ composta por psicólogos com menos de cinco anos de atividades na rede federal de ensino. A maioria dos sujeitos (70\%) concluíram algum curso de pós-graduação (stricto sensu ou lato sensu) entre os anos de 2000 e 2009 e um total de 12,2\% realizou outra graduação além do curso de Psicologia. $\mathrm{O}$ total de 78,5\% dos psicólogos atua em campi situados na zona urbana, e na maioria dos casos o psicólogo é o único profissional da área nessa instituição (79\%), com nomeação de 40 horas semanais de trabalho $(97,6 \%)$. Observou-se um percentual acima de $20 \%$ dos profissionais que exercem outras atividades remuneradas, tais como docência e atividades de psicologia clínica.

\section{Instrumento}

Inicialmente foi desenvolvido um questionário sobre a Autoeficácia para Psicólogos em Contexto Escolar, contendo 35 questões, com enfoque na capacidade do psicólogo em exercer suas atividades nas diferentes áreas possíveis de intervenção (áreas que podem ser agrupadas) As áreas foram identificadas com base na literatura, nomeadas em algumas pesquisas e estudos de caraterização dos perfis desses profissionais (Jimerson, 2016; Matteucci \& Coyne, 2017; Peretta et al., 2014; Walcott \& Hyson, 2018). Assim, foram elaboradas questões que considerassem aspetos gerais da atuação do psicólogo em contexto escolar; intervenção individual, tanto em nível de avaliação (com utilização dos instrumentos) quanto de escuta psicológica; intervenção no contexto escolar, relação com as famílias, relações com outros profissionais, tanto docentes quanto outros técnicos e colaboração e comunicação com chefias.

Para além de um levantamento das ações do psicólogo, para a elaboração dos itens consultou-se um conjunto de escalas de autoeficácia geral e específica, dirigidas a profissionais de outros contextos (Pedrazza, Trifiletti, Berlanda \& Bernardo, 2013; Polydoro \& GuerreiroCasanova, 2010; Schaufeli, Salanova, González-Romá \& Bakker, 2002). Além disso, a redação dos itens e a estrutura geral buscaram respeitar o referencial teórico de base da TSC e as indicações subjacentes para a construção de instrumentos de autoeficácia.

Após elaborar e revisar o questionário, surgiu a primeira versão do que foi denominado como Escala de Autoeficácia para Psicólogos em Contexto Escolar: a EAPsi. Essa versão foi enviada para que especialistas analisassem a pertinência e a abrangência das áreas e ações contempladas, assim como a clareza na formulação dos itens. Foram escolhidos cinco especialistas/psicólogos atuantes no contexto educacional para realizar essa análise, e a escolha desses profissionais embasou-se nos seguintes critérios: 1. estar atuando como psicólogo em contexto escolar (exceto docentes da graduação); 2. ter realizado pós-graduação na área da psicologia escolar/ educacional (no mínimo mestrado, mas preferencialmente doutorado). Os especialistas fizeram poucas sugestões de alterações, especificamente na formulação dos itens e na clareza na redação.

Essa $1^{\text {a }}$ versão da EAPsi foi aplicada, concomitantemente, no Brasil e em Portugal. Para tanto, foi realizado um convite via e-mail aos profissionais e órgãos de gestão, fornecendo o link de acesso ao questionário. 
Ele continha 35 itens, que foi aplicada a 62 psicólogos atuantes em contexto escolar no Brasil (Koehler \& Mata, 2019) e a 296 dos profissionais em Portugal (Mata, Santos \& Koehler, 2018). Na ocasião, acrescentou-se um campo específico para comentários que os respondentes considerassem pertinentes, diante do instrumento ou de suas respostas. O objetivo era coletar informações que pudessem subsidiar modificações, caso necessário. Com base no tratamento estatístico exploratório e na análise de comentários, algumas alterações foram realizadas, tanto em nível da escala de resposta, quanto de formulação dos itens. Em relação à escala de resposta, foi utilizada inicialmente a de tipo Likert, de 4 pontos, mas a possibilidade de respostas foi ampliada de 1 (discordo totalmente) a 10 (concordo totalmente).
$\mathrm{Na}$ formulação dos itens, não apenas a formulação específica de alguns foi alterada, como também a expressão "sou capaz", que foi substituída pelo termo "consigo", pois a concepção semântica, e consequentemente sua interpretação levantou muitas dúvidas. Também foi alterada a indicação geral dada no início do questionário, onde se lia “[...] são apresentadas algumas afirmações relacionadas com a forma como se podem sentir psicólogos em contexto escolar[...]", após a modificação passou a ler-se: [...] você encontra algumas tarefas que podem ser desenvolvidas por um psicólogo em contexto escolar. Indique até que ponto na sua atividade profissional, no contexto e situação atuais, sente que as consegue desenvolver. Os itens dessa versão são apresentados na Tabela 1.

Tabela 1

Itens da Escala de Autoeficácia para Psicólogos a trabalhar em Contexto Escolar EAPsi

Itens

1. Consigo comunicar-me de maneira eficaz com a equipe gestora.

2. Consigo arranjar canais de comunicação eficazes quando necessito comunicar-me com as famílias.

3. Consigo atingir meus objetivos de psicólogo na instituição onde trabalho.

4. Quando necessário, consigo avaliar a eficácia das minhas intervenções.

5. Consigo colaborar com a equipe gestora.

6. No percurso do ano letivo, é fácil para mim contribuir na formação da equipe pedagógica.

7. Consigo comunicar-me adequadamente com meus colegas de trabalho.

8. Consigo gerenciar os compromissos relacionados à minha atividade profissional.

9. Consigo responder as necessidades individuais dos alunos da instituição.

10. Consigo desenvolver minhas obrigações, sem ser cobrado pela equipe gestora.

11. Consigo elaborar pareceres e laudos psicológicos, e realizo registros dos atendimentos.

12. Consigo encaminhar casos para outros profissionais, quando necessário.

13. Consigo estabelecer relações positivas com colegas do meu trabalho de modo a ter um bom relacionamento na instituição.

14. Consigo realizar avaliações psicológicas quando necessárias no contexto da escola.

15. Consigo executar ações de intervenção junto às turmas.

16. Graças ao meu desempenho eu consigo manter-me atualizado para melhor executar as minhas funções no trabalho.

17. Consigo ofertar atendimento a pais e demais familiares dos alunos.

18. Consigo investir o esforço necessário para identificar as principais necessidades de intervenções individuais no contexto onde trabalho.

19. Consigo opinar a respeito do que deve ser feito no trabalho da equipe onde atuo.

20. Consigo colaborar na organização de ações socioeducativas (ações de sensibilização, palestras, oficinas, cursos) para a comunidade escolar.

21. Consigo pensar em alternativas para resolver problemas que surgem no meu trabalho.

22. Consigo participar do planejamento da política educacional da instituição.

23. Consigo pensar e executar ações relacionadas ao desenvolvimento dos alunos.

24. Consigo enfrentar adversidades do meu trabalho.

25. Consigo procurar apoio da equipe gestora quando necessário.

26. Consigo planejar a curto, médio e longos prazos o que deve ser feito no meu trabalho.

27. Consigo promover o envolvimento das famílias no processo educativo.

28. Consigo propor atividades em grupo para responder a problemáticas identificadas.

29. Consigo realizar diagnóstico, análise e intervenção em nível institucional.

30. Consigo resolver problemas difíceis no meu trabalho.

31. Consigo responder aos pedidos de trabalho feitos pela equipe gestora.

32. Consigo definir o (s) instrumento (s) de avaliação/caracterização mais adequado (s) para cada caso.

33. Consigo trabalhar em equipe.

34. Consigo promover uma boa relação escola família maximizando o potencial de colaboração de cada família.

35. Consigo utilizar os instrumentos/provas necessários para a avaliação e caraterização dos casos e situações com que me deparo. 


\section{Procedimentos de Coleta de Dados}

Tendo realizado o estudo piloto da EAPsi, procedeu-se a aplicação do questionário alongado. Inicialmente, realizou-se a busca do público-alvo. É necessário ressaltar que o questionário aplicado nessa ocasião continha sete seções, por tratar-se da pesquisa do doutoramento em Psicologia. Neste artigo, somente as questões relativas à autoeficácia serão analisadas.

Inicialmente foi realizada a coleta dos e-mails dos psicólogos atuantes nos IFs, com a colaboração dos servidores da área de recursos humanos e dos profissionais ligados ao ensino dessas instituições. Por se tratar de uma participação anônima e voluntária, os psicólogos autorizaram a utilização do e-mail institucional, que serviu como veículo de comunicação para apresentar e fomentar a participação desses profissionais. Em segui$\mathrm{da}$, foi enviado um segundo $e$-mail contendo a pesquisa: "A Identidade dos Psicólogos dos Institutos Federais de Educação, Ciência e Tecnologia”, que ficou armazenada no Google Docs. Nesse instrumento foram explicitados os objetivos da pesquisa e os seus critérios éticos: garantia de sigilo; participação voluntária, mas específica dos psicólogos dos Institutos Federais de Educação; ausência de ônus e bônus; e a utilização dos dados para análises e publicações. Somente quem concordasse com esses critérios teria acesso ao questionário. A primeira anuência foi o termo de Consentimento Livre e Esclarecido (TCLE), já que somente após aceitá-lo o participante poderia responder ao questionário.

A pesquisa ficou disponível para respostas durante seis meses, entre outubro de 2017 a março de 2018. Nesse período foram realizadas várias ações para atrair a participação dos psicólogos, como o reenvio dos e-mails (Regmi, Waithaka, Paudyal, Simkhada, \& van Teijlingen, 2016) e a divulgação da pesquisa na página do Facebook (psicólog@s - institutos federais), criada em 2013 pelos profissionais atuantes nos Institutos Federais de Educação.

Os procedimentos de recolha foram validados pela Comissão de Ética do Centro de Gestão da Investigação, do ISPA-Instituto Universitário.

\section{Análise de dados}

Depois de finalizada a coleta dos dados, foi realizada a análise fatorial exploratória (AFE) com o uso do programa FACTOR 10.9.01 (Ferrando \& Lorenzo-Seva, 2006, 2019), a fim de definir a estrutura fatorial método Robust Unweighted Least Squaares (RULS), utilizando uma matriz de correlações policóricas como recomendado para a análise fatorial de escalas de itens ordinais de tipo Likert (Asún, Rdz-Navarro \& Alvarado, 2015; Holgado-Tello, Chacón-Moscoso, Barbero-García \& Vila-Abad, 2010). Uma vez que esperávamos que as dimensões avaliadas estivessem correlacionadas, foi utilizado o método de rotação oblíqua promin Lorenzo.

Assim, a estrutura fatorial da Escala de Autoeficácia para Psicólogos foi analisada por meio de uma AFE. Para reter apenas o número mínimo de fatores para explicar convenientemente a autoeficácia dos psicólogos em contexto escolar, foi utilizado o critério Kaiser-Guttman como ponto de partida. No entanto, uma vez que esse critério de Kaiser-Guttman tende a superestimar o número de fatores retidos, recorreu-se ao método das análises paralelas (APs) para confirmar esses resultados. Neste método os eigenvalues obtidos pelo critério KaiserGuttman são comparados (pareados) com o percentil 95 dos eigenvalues determinados aleatoriamente num conjunto hipotético de 500 matrizes de correlação de variáveis. Assim, os fatores com eigenvalues superior a 1, mas com eigenvalues menores que os calculados nas APs são descartados (Damásio, 2012). Finalmente, para determinar se os modelos fatoriais sugeridos representam corretamente os dados, foram calculados os seguintes índices de ajustamento (utilizando Bootstrap para análises mais robustos), cujos valores de corte sugeridos (Schreiber, Nora, Stage, Barlow, \& King, 2006) também apresentamos: rácio do qui-quadrado pelos graus de liberdade $\left(\chi^{2} / d f \leq 2\right.$ ou 3); Comparative Fit Index (CFI $\left.\geq, 95\right)$; Tucker-Lewis Index (TLI $\geq$,96) e Root Mean Square Error Of Approximation (RMSEA $<, 06$ a ,08 com intervalo de confiança).

\section{Resultados}

De acordo com o critério de Kaiser-Guttman, os resultados da AFE, realizados com os 35 itens da Escala de Autoeficácia para Psicólogos, sugeriram a extração dos oito fatores que explicam $69,14 \%$ da variância. No entanto, apenas três fatores apresentaram autovalores superiores ao percentil 95 dos autovalores obtidos numa análise paralela com 500 matrizes correlacionais aleatórias, sugerindo a retenção de três fatores, que explicam $51,1 \%$ da variância.

Contudo, observou-se que vários itens $(3,6,7,9$, $13,18,23,28,31,33$, e 35 ) apresentavam cargas fatoriais superiores a $0,32 \mathrm{em}$ mais de um fator, com uma diferença menor a 0,2 entre as cargas de ambos os fatores. Após a remoção destes itens, tal como recomendado por Howard (2016) foi definido um novo modelo fatorial com 24 itens para os quais a AP sugeriu a retenção de três fatores que explicavam $53,72 \%$ da variância. Nesse modelo, o item 10 apresentou cargas fatoriais interiores a 0,32 . Além disso, o fator 2 , incluiu um item que, conceitualmente, não estava relacionado aos itens restantes (a afirmativa 15 não se relaciona com a intervenção com a família como o restante dos itens). Assim, estes dois itens foram também retirados da análise.

O novo modelo fatorial, com 22 itens, apresentou autovalores que sugeriram a retenção de cinco fatores que explicavam $65,1 \%$ da variância. A AP sugere reter novamente três fatores, uma vez que só os autovalores dos três primeiros fatores foram superiores ao percentil 95 dos autovalores aleatórios calculados (Tabela 2). 
Assim, adotou-se uma estrutura fatorial com três fatores que explicam $55,1 \%$ da variância. $\mathrm{Na}$ Tabela 2, resumem-se as cargas fatoriais de cada item para cada um dos fatores e para consistência interna, avaliada pelo alfa de Cronbach. O modelo é considerado adequado em virtude de apresentar índices de ajustamento excelentes $\left(\chi^{2}(168)=122,92 ; p=0,996 ; \quad \mathrm{CFI}=0,997 ; \mathrm{TLI}=0,996\right.$; RMSEA $=0,024, \operatorname{IC} 95 \%[0,015 ; 0,021])$.

Tabela 2

Cargas Fatoriais, Comunalidades e Estatísticas Descritivas da Escala de Autoeficácia para Psicólogos em Contexto Escolar (22 itens)

\begin{tabular}{|c|c|c|c|c|}
\hline \multirow{2}{*}{ Itens } & \multicolumn{3}{|c|}{ Fator } & \multirow{2}{*}{ Comunalidades } \\
\hline & 1 & 2 & 3 & \\
\hline 4 & ,909 &,- 112 &,- 110 &, 596 \\
\hline 26 & ,804 & ,115 &,- 205 & ,582 \\
\hline 32 & ,701 &,- 284 & ,189 & ,539 \\
\hline 12 & 659 &,- 162 & ,123 & ,422 \\
\hline 21 & 637 & ,134 &,- 013 &, 502 \\
\hline 16 & ,590 &,- 197 & ,104 & ,421 \\
\hline 29 & ,585 & ,094 &,- 064 & ,363 \\
\hline 30 & ,577 & ,221 &,- 025 & ,496 \\
\hline 14 & ,553 &,- 259 & ,040 & ,256 \\
\hline 11 & ,535 & ,067 & ,113 & ,301 \\
\hline 8 & ,514 & ,723 & ,086 & ,371 \\
\hline 24 & ,459 & ,209 &,- 101 & ,297 \\
\hline 20 & ,352 & ,298 & ,041 & ,348 \\
\hline 1 &,- 172 & ,901 & ,107 & ,713 \\
\hline 25 &,- 031 & ,862 &, 034 & ,730 \\
\hline 5 & ,037 & ,723 & ,090 & ,596 \\
\hline 19 & ,256 & ,516 & ,032 & ,490 \\
\hline 22 & ,152 & ,477 & ,057 & ,354 \\
\hline 34 &,- 157 & ,027 & 937 & ,727 \\
\hline 27 &,- 016 & ,031 & ,832 & ,688 \\
\hline 17 &,- 040 &,- 025 & ,777 & ,618 \\
\hline 2 & ,089 & ,215 & ,446 & ,369 \\
\hline Autovalor empírico & 8,181 & 2,393 & 1,544 & \\
\hline Autovalor aleatório & 1,760 & 1,608 & 1,506 & \\
\hline \% Variância & 37,19 & 10,88 & 7,02 & \\
\hline Alfa & ,86 & ,84 &, 84 & \\
\hline Média & 7,12 & 7,02 & 6,64 & \\
\hline DP & 1,30 & 1,81 & 1,95 & \\
\hline Sk & $-0,51$ & $-0,86$ & $-0,94$ & \\
\hline $\mathrm{Ku}$ & 0,07 & 0,52 & 0,50 & \\
\hline \multicolumn{5}{|l|}{ Correlações (r) } \\
\hline Factor 2 & .51 & & & \\
\hline Factor 3 & .52 & .35 & & \\
\hline
\end{tabular}

Nota. Método de Extração: Robust Unweighted Least Squares (RULS); Método de Rotação: Promin; a=O Percentil 95 dos eigenvalues aleatórios foram calculados com base em uma matriz hipotética aleatória faturada 500 vezes, para 22 itens e $n=205$

No fator 1, que explica $37,2 \%$ da variância, saturaram treze itens $(4,26,32,12,21,16,29,30,14,11,8$, 24 e 20) relacionados com a Autoeficácia Profissional como psicólogo, tanto no nível de intervenção individual (e.g., item 4 - "Quando necessário, consigo avaliar a eficácia das minhas intervenções”), tanto a nível de avaliação com utilização dos instrumentos (e.g., item 32 - "Consigo definir o(s) instrumento (s) de avaliação/caracterização mais adequado (s) para cada caso"), quanto de intervenção escolar (e.g., item 20 - "Consigo 
colaborar na organização de ações socioeducativas para a comunidade escolar"). O segundo fator, que explica $10,88 \%$ da variância, está composto por 5 itens (1, 25, 5,19 , e 22) relacionados com a Autoeficácia Relacional, por exemplo: "Consigo procurar apoio da equipe gestora quando necessário"; "Consigo opinar a respeito do que deve ser feito no trabalho da equipe onde atuo". Finalmente, no fator 3 , saturaram quatro itens $(34,27,17$ e 2) relacionados com a Autoeficácia na articulação com as famílias os quais explicam 7,02\% de variância (e.g., "Consigo promover uma boa relação escola-família maximizando o potencial de colaboração de cada família"; "Consigo promover o envolvimento das famílias no processo educativo"). Esses três fatores apresentaram bons níveis de consistência interna (ver Tabela 2).

Foram calculados os escores de cada fator por meio da média dos itens que o conformam, e as suas respetivas estatísticas descritivas são apresentadas na Tabela 2. As correlações entre os fatores são moderadas. Para identificar se os psicólogos apresentam diferentes níveis de autoeficácia, dependendo das áreas e ações de atuação, calculou-se o escore de cada subescala/fator as médias e realizou-se a comparação através de uma Análise de Variância (ANOVA) de medidas repetidas. Os resultados indicam que existem diferenças significativas entre os fatores avaliados pela Escala de Autoeficácia para Psicólogos em Contexto escolar: $(F(1,74 ; 354,43)=7,88$, $\left.p=0,001, \eta p^{2}=0,04, \pi=0,93\right)$, indicando que os psicólogos apresentam diferentes níveis de autoeficácia dependendo do tipo de ação ou intervenção à qual se refere. Foram utilizadas comparações Pairwise com correção de Bonferroni para identificar entre quais escalas existem diferenças significativas. De acordo com essas comparações, foram observados valores significativamente mais baixos na Autoeficácia na articulação com a família quando comparada aos outros dois fatores $(p<0,001$ quando comparado com a Autoeficácia Profissional e $p=0,037$ com a Autoeficácia Relacional).

\section{Discussão}

Pretendeu-se com esse estudo apresentar o desenvolvimento da validação de uma Escala de Autoeficácia para psicólogos que atuam em contexto escolar. Devido especialmente ao aumento de profissionais que atuam nesse contexto, associado às alterações sofridas a vários níveis, a atuação desses psicólogos têm-se tornado mais multifacetada, não só sobre os públicos-alvo de intervenção (e.g., famílias, colegas de trabalho, equipe gestora) como no tipo e estratégias de intervenção necessárias para responder às necessidades dos contextos (Bell \& McKenzie, 2013; Graves, Proctor \& Aston, 2014). Desse modo a escala poderá ser utilizada na investigação, como também na prática dos profissionais em contexto escolar. Uma vez que construto de autoeficácia é específico e relativo a diversos domínios (Bandura, 1977; Bandura,
Azzi \& Polydoro, 2008; Nunes \& Noronha, 2008), e a autoeficácia é importante para compreender processos motivacionais, o desempenho, a qualidade da ação educativa e o bem-estar (Fonsêca et al., 2018; Holzberger et al., 2013; Huang, 2016; Höltge et al., 2017; Kim \& Seo, 2018; Poulou et al., 2018; Skaalvik \& Skaalvik, 2014; Street, Malmberg, \& Stylianides, 2017; Talsma et al., 2018), o desenvolvimento dessa escala apresenta-se como necessária e útil, tanto na investigação, como também na prática dos profissionais em contexto escolar.

O processo de construção da escala, que foi designada EAPsi, sustentou-se, inicialmente na revisão da literatura e análise de alguns instrumentos para públicos em específicos. Já nesse momento, percebia-se a dificuldade de encontrar produções direcionadas para os psicólogos em contexto escolar. Posteriormente, a participação dos especialistas assegurou a validação do conteúdo.

Os profissionais que participaram desse estudo foram psicólogos dos Institutos Federais, pela facilidade de acesso, associado ao foco de estudos de um trabalho mais amplo, como pelo fato do seu contexto de trabalho que contempla as diferentes áreas de intervenção e exigências estratégicas e de competências que se colocam aos psicólogos a trabalhar em contexto escolar atualmente (Brown, 2016; Feitosa, 2017; Graves et al., 2014; Marinho-Araujo \& Almeida, 2014; Van Overschelde \& Lasser, 2019). Esses locais são espaços privilegiados, pois contam com diversos profissionais e inúmeros docentes que facilitam o desenvolvimento de ações acrescido a baixa rotatividade de pessoal. Assim sendo, entende-se como um importante espaço para a concretização do papel do psicólogo em contexto escolar.

A formação desses locais de trabalho está relacionada à expansão da Educação Profissional na Rede Federal de Ensino que construiu, desde 2003, mais de 500 novas unidades de ensino, levando à nomeação de psicólogos para comporem a equipe multidisciplinar dos campi. Apesar de se tratar de uma atuação recente nesses locais e em número significativo nessa modalidade de ensino, é possível afirmar que as ações desses profissionais auxiliaram para o bom funcionamento das instituições, alcançando espaços antes negligenciados. No entanto, compor o quadro de servidores federais ligados ao ensino não é sinônimo de "formar" novos espaços e, sim, o compromisso que os profissionais assumem de desenvolver ações para o fortalecimento dos processos de ensino-aprendizagem, da transformação social, da conscientização e do empoderamento da sua comunidade acadêmica e dos diversos autores desse contexto (Marinho-Araujo \& Almeida, 2014).

A validade do construto estudou-se pela análise fatorial exploratório, com o método de estimação Robust Unweighted Least Squares (RULS), com rotação promin, sendo eliminados 13 itens, ficando a versão final da EAPsi composta por 22 afirmações. A solução final de três fatores explica $55,1 \%$ do total da variância da escala e 
apresenta um ajustamento excelente aos dados. A versão final revelou também bons valores de consistência interna para todas as escalas.

O instrumento que foi elaborado partiu então dessas diferentes áreas e competências de atuação profissional dos psicólogos que têm vindo a ser apresentadas na literatura. Desses diferentes focos de intervenção contemplados nos itens elaborados, após a AFE extraíram-se três fatores. Em um primeiro fator surgiram os itens relativos a autoeficácia profissional que se refere as ações que o psicólogo demonstra nas atitudes diárias. Estão relacionadas às ações individuais que o psicólogo demonstra na instituição, tais como busca de soluções a problemas diversos, enfrentamento de adversidades, colaboração com os colegas de trabalho, escolha e uso de testes psicológicos, entre outros. Sinteticamente, são ações individuais nas quais o profissional avalia sua intervenção como à atualização de conhecimentos e até resolução de problemas e os desafios que são colocados na prática, além do manuseio de instrumentos de avaliação, levando em conta que os profissionais relatam gastar até $50 \%$ do seu tempo envolvido na avaliação psicoeducacional (Bowles, Scull, Clinton \& Larkins, 2016).

O segundo fator destacou-se a autoeficácia nas relações com a direção e gestão da instituição, onde os diferentes itens contemplavam não só aspetos relacionais com a equipe gestora como também a intervenções no âmbito da política institucional. Essa dimensão é central para se compreender a atuação institucional do psicólogo, uma vez que a equipe gestora parece ter influência sobre as capacidades em realizar atividades e alcançar resultados específicos, associado ao papel de assessoria que o psicólogo deve desenvolver na instituição escolar. O estar inserido, como membro integrante e participantes da equipe gestora parece ser um novo aspecto a ser considerado na atuação do psicólogo escolar (Chiocchio, Kelloway \& Hobbs, 2015; Marinho-Araujo \& Almeida, 2014; Petroni \& Souza, 2014).

A terceira dimensão identificada foi a relativa à intervenção junto as famílias. Sabe-se da necessidade de se envolverem as famílias como parceiros no processo educativo dos filhos e dos benefícios daí decorrentes podendo os psicólogos serem elementos centrais na articulação e no apoio à construção dessas parcerias (Bahr et al., 2017; Epstein, Sanders, Sheldon \& Simon, 2018; Vega, Lasser \& Fernandez, 2017). Os itens que integram essa dimensão contemplam dois aspetos centrais identificados na literatura: a comunicação e as estratégias para promover uma participação efetiva de famílias com caraterísticas e necessidades diversificadas. As famílias devem partilhar objetivos e responsabilidades de modo a promover o seu envolvimento e uma parceria implica arranjos cooperativos nos quais a informação e os recursos fluem nos dois sentidos, em contraste com a tradição de comunicação unidirecional da escola para a casa (Filter, Ebsen \& Dibos, 2013; Reschly \& Christenson,
2019; Vega et al., 2017). Assim sendo, os resultados deste estudo fornecem evidências iniciais de que as propriedades psicométricas do EAPsi são adequadas, sugerindo que a escala é um instrumento aceitável para avaliar a autoeficácia dos psicólogos que atuam em contexto escolar.

Levando em conta que os resultados deste estudo, bem como a utilização da escala e o planejamento de novas pesquisas, devem ser considerados à luz de algumas limitações. Um dos aspetos a ser considerado neste estudo é o público-alvo, limitado aos psicólogos escolares dos Institutos Federais de Educação. Apesar da participação ter sido próximo a 50\%, o número de sujeitos, para validação dessa escala esse valor não é ideal. Faz-se necessário conhecer com maior profundidade os psicólogos que atuam nos demais contextos escolares (escolas de ensino fundamental, escola de ensino médio, instituições públicas municipais e estaduais, como também as instituições privadas e organizações não governamentais) de forma a diversificar a ampliar o público da amostra. Somente assim será possível melhorar o conhecimento sobre autoeficácia profissional dos psicólogos em contexto escolar, pois tal construto relaciona-se com a escolha de estratégias, a persistência perante dificuldades, forma de lidar com situações estressantes, processos motivacionais, desempenho e qualidade da ação (Bandura, 2008; Bandura et al., 2008; Cardoso \& Baptista, 2018; Fonsêca et al., 2018; Huang, 2016; Shoji et al., 2016). Perante essa centralidade tem surgido a necessidade de intensificar pesquisas que visem à construção de instrumentos específicos para profissionais que se enquadrem nas suas atividades e contextos particulares. Assim ampliar o público de participação tornará a pesquisa mais fidedigna em futuros trabalhos.

Todavia, esses primeiros dados demonstram que a escala avalia a autoeficácia dos psicólogos em contexto escolar, parecem reveladores da utilidade da mesma. A construção dessa escala pode responder a lacunas existentes, uma vez que a sua utilização isolada ou associada a outros instrumentos, pode ser útil para a investigação do papel dos psicólogos atuantes em contexto escolar.

A autoeficácia é um construto com grande apoio teórico e empírico. Para criar essa escala, teve de ser explorado o domínio do funcionamento e as ações que os psicólogos realizam rotineiramente em suas atividades. Ressalta-se ainda a intenção de se prosseguir com estudos de validação dessa escala, estendendo a diversidade da amostra, que nesse caso, também, esteve voltada principalmente para a brasileira, e promovendo a comparação com critérios externos de validade. Assim, parece oportuno o investimento em estudos que busquem conhecer a realidade nacional e, especificamente, o comportamento da AEPsi. Além disso, pode-se ampliar o estudo para verificar a relação entre os diversos contextos escolares: público, privado, ensino fundamental e médio, de modo a 
abranger o conjunto de informações sobre a relação entre a percepção da autoeficácia, ambiente e comportamento, como proposto no modelo de reciprocidade triádica da Teoria Social Cognitiva (Bandura, 2012).

\section{Considerações Finais}

Focar os estudos nos papéis que o psicólogo escolar assume nas instituições de ensino apresenta-se como uma necessidade urgente, tendo em vista, as mudanças que estão ocorrendo nesse contexto. Norteados por estes fundamentos, procedemos à construção e validação de uma Escala de Autoeficácia para Psicólogos em Contexto Escolar (EAPsi) que apresentou as dimensões de diferentes áreas de atuação que o profissional tem desenvolvido. Embora possa existir outros instrumentos específicos
(Guiney et al., 2014), orientavam-se para avaliar aspetos recentemente incluídos nas atividades do psicólogo escolar, em especial sua participação na gestão da instituição e no engajamento em intervenções a nível de sistema (Bahr et al., 2017), razões que sustentaram o desenvolvimento deste estudo. A versão final da AEPsi ficou constituída por 22 afirmações, organizadas em três subescalas que se ajustam ao significado dos itens que as constituem e ao construto a medir, o que representa uma mais-valia do ponto de vista da validade da escala. Apesar das boas indicações da EAPsi, quanto às suas propriedades psicométricas, futuras aplicações são necessárias. Assim, sugere-se que novos estudos sejam desenvolvidos e possam contribuir para a melhoria da robustez dessa escala, que pode ser uma ferramenta útil na prática dos profissionais psicólogos em contexto escolar.

\section{Referências}

Almeida, L. S., \& Peixoto, F. (2011). Psicologia da Educação. Em M. P. Lopes, P. J. Palma, R. B. Ribeiro, \& M. E. Pina e Cunha (Eds.), Psicologia Aplicada (pp. 47-68). Lisboa: RH.

Ambiel, R. A., \& Noronha, A. P. P. (2012). Autoeficácia para escolha profissional: Teoria, pesquisas e avaliação. Psicologia em Pesquisa, 6(2), 171-178. doi:10.5327/Z1982-12472012000200010

Asún, R. A., Rdz-Navarro, K., \& Alvarado, J. M. (2015). Developing multidimensional likert scales using item factor analysis: The case of four-point items. Sociological Methods E Research, 45(1), 109-133. doi:10.1177/0049124114566716

Azzi, R. G., \& Vieira, D. (2014). Crenças de eficácia em contexto educativo. São Paulo: Casa do Psicólogo.

Bahr, M. W., Leduc, J. D., Hild, M. A., Davis, S. E., Summers, J. K., \& McNeal, B. (2017). Evidence for the expanding role of consultation in the practice of school psychologists. Psychology in the School, 54(6), 581-595. doi:10.1002/pits.22020

Bandura, A. (1977). Self-efficacy: Toward a unifying theory of behavioral change. Psychological Review, 84, 191-215. doi:10.1037/0033295X.84.2.191

Bandura, A. (1986). Social foundations of thought and action: A social cognitive theory. NJ:Prentice Hall.

Bandura, A. (1995). Self-efficacy in changing societies. New York: Cambridge University Press.

Bandura, A. (1997). Self-efficacy: The exercise of control. New York: Worth Publishers.

Bandura, A. (2001). Social Cognitive Theory: An agentic perspective. Annual Review Psychology, 52, 1-26.

Bandura, A. (2008). A evolução da teoria social cognitiva. Em A. Bandura, R. Azzi, \& S. Polydoro (Eds.), Teoria Social Cognitiva: conceitos básicos (pp. 15-41). Porto Alegre: Artmed.

Bandura, A. (2012). On the functional properties of perceived self-efficacy revisited. Journal of Management, 38, 9-44. doi:10.1177/0149206311410606

Bandura, A., Azzi, R. G., \& Polydoro, S. (2008). Teoria Social Cognitiva: Conceitos básicos. Porto Alegre: Artmed.

Bell, H., \& McKenzie, V. (2013). Perceptions and realities: The role of school psychologists in Melbourne, Australia. The Australian Educational and Developmental Psychologist, 30, 54-73. doi:10.1017/edp.2013.1

Bertollo-Nardi, M. (2014). O trabalho do psicólogo em um campus do Instituto Federal do Espírito Santo: possibilidade e desafios de uma prática. (Tese de Doutorado). Universidade Federal do Espírito Santo, Vitória- ES. (B546t)

Bisinoto, C. E. O., \& Marinho-Araujo, C. M. (2009). Psicologia escolar: Cenários atuais. Estudos e Pesquisas em Psicologia, 9(3), 648-663. Recuperado de http://search.ebscohost.com/login.aspx?direct $=$ true\&db=edssci\&AN=edssci.S1808.42812009000300007〈=ptbr\&site $=$ eds-live $\&$ scope $=$ site

Bowles, T., Scull, J., Clinton, J., \& Larkins, G. (2016). Conducting psychological assessments in schools: Adapting for converging skills and expanding knowledge. Issues in Educational Research, 26(1), 10-28. doi:020531978552133

Brown, J. A., Watanabe, Y., Lee, D. H., McIntosh, K. (2016). School psychology research and practice in East Asia: Perspectives on the past, present, and future directions of the field. School Psychology International, 37(6), 557-582. doi:10.1177/0143034316671354

Cardoso, H. F., \& Baptista, M. N. (2018). Escala de autoeficácia no trabalho: Evidências de validade e precisão. Avaliação Psicológica, 17(2), 188-198. doi:10.15689/ap.2018.1702.13946.04

Cavalcante, M. M., Siqueira, M. M. M., \& Kuniyoshi, M. S. (2014). Engajamento, bem-estar no trabalho e capital psicológico: Um estudo com profissionais da área de gestão de pessoas. Revista Pensamento e Realidade, pp. 42-63.

Chiocchio, F., Kelloway, K., \& Hobbs, B. (2015). The psychology and management of project teams. Oxford University Press.

Consiglio, C., Borgogni, L., Tecco, C., \& Schaufeli, W. (2016). What makes employees engaged with their work? The role of selfefficacy and employee's perceptions of social context over time. Career Development International, 21(2), 125-143. doi:10.1108/ CDI-03-2015-0045

Damásio, B. F. (2012). Uso da análise fatorial exploratória em psicologia. Avaliação Psicológica, 11(2), 213-228. Recuperado de http://pepsic. bvsalud.org/pdf/avp/v11n2/v11n2a07.pdf 
Epstein, J., Sanders, M., Sheldon, S., \& Simon, B. (2018). School, Family, and Community Partnerships: Your Handbook for Action. Washington, DC.: A Sage Publications Company.

Fagan, T. (1992). Compulsory schooling, child study, clinical psychology, and special educacion: Origins of school psychology. American Psychologist, 47, 236-243. doi: 10.1037//0003-066x.47.2.236

Feitosa, L. R. C. (2017). Psicologia Escolar nos Institutos Federais de Educação, Ciência e Tecnologia: Contribuições para a atuação na educação superior (Tese de Doutorado). Universidade Federal de Brasília, Brasília.

Ferrando, P. J., \& Lorenzo-Seva, U. (2006). FACTOR: A computer program to fit the exploratory factor analysis model. Behavior Research Methods, 38(1), 88-91. doi:10.3758/BF03192753

Ferrando, P. J., \& Lorenzo-Seva, U. (2019). On the added value of multiple factor score estimates in essentially unidimensional models. Educational and Psychological Measurement, 79(2), 249-271. doi:10.1177/0013164418773851

Filter, K. J., Ebsen, S., \& Dibos, R. (2013). School psychology crossroads in America: Discrepancies between actual and preferred discrete practices and barriers to preferred practice. International Journal of Special Education, 28(1), 88-100.

Fonsêca, P., Couto, R., Silva, M. I. F., Coelho, G. L. H., \& Carvalho, T. A. (2018). Adaptação brasileira do Inventario de Autoeficacia para el Estudio. Revista Psicologia: Teoria e Prática, 20, 60-71. doi:10.5935/1980-6906/psicologia.v20n3p60-71

Frigotto, G. o. (2018). Institutos Federais de Educação, Ciência e Tecnologia: Relação com o ensino médio integrado e o projeto societário de desenvolvimento. Em L. UERJ (Ed.), (pp. 320).

Graves, S., Proctor, S., \& Aston, C. (2014). Professional roles and practices of school psychologists in urban schools. Psychology in the Schools, 51(4), 384-394. doi:10.1002/pits

Guerreiro-Casanova, D. C. F., L. C. M. \& Azzi, R. G. (2015). Autoeficácia acadêmica de estudantes de Ensino Médio: Especificidades das séries e turnos. Psicologia Ensino \& Formação, 6(1), 95-120. Recuperado de http://pepsic.bvsalud.org/scielo.php?script=sci_arttext\&pid $=$ S2177-20612015000100007

Guiney, M. C., Harris, A., Zusho, A., \& Cancelli, A. (2014). School psychologists' sense of self-efficacy for consultation. Journal of Educational and Psychological Consultation, 24(1), 28-54. doi:10.1080/10474412.2014.870486

Guzzo, R. (2015). Critical psychology \& american continent: From colonization and domination to liberation and emancipation. Em I. O. Parker (Ed.), Handbook of critical psychology (pp. 406-414).

Helgoth, S., \& Sobansky, R. (2008). The roles and functions of school psychologists: A global perspetive. International Journal of Psychology: A Biopsychosocial Approach, 1, 85-98. doi:20.500.12259/32375

Holgado-Tello, F. P., Chacón-Moscoso, S., Barbero-García, I., \& Vila-Abad, E. (2010). Polychoric versus Pearson correlations in exploratory and confirmatory factor analysis of ordinal variables. Quality \& Quantity, 44, 153. doi:10.1007/s11135-008-9190-y

Holzberger, D., Philipp, A., \& Kunter, M. (2013). How teachers' self-efficacy is related to instructional quality: A longitudinal analysis. Journal of Educational Psychology, 105(3), 774-786. doi:10.1037/a0032198

Howard, M. C. (2016). A review of exploratory factor analysis decisions and overview of current practices: What we are doing and how can we improve? International Journal of Human-Computer Interaction, 32(1), 51-62. doi:10.1080/10447318.2015.1087664

Huang, C. (2016). Achievement goals and self-efficacy: A meta-analysis. Educational Research Review, 19, 119-137. doi:10.1016/j. edurev.2016.07.002

Höltge, L., Ehm, J., Hartmann, U., \& Hasselhorn, M. (2017). Teachers' self-efficacy beliefs regarding assessment and promotion of schoolrelevant skills of preschool children. Early Child Development and Care, 189(2), 339-351. doi:10.1080/03004430.2017.1323888

Jimerson, S. R. (2016). Enhancing science, practice, and policy relevant to school psychology around the world. School Psychology Quarterly, 31(1), 1-7. doi:10.1037/spq0000153

Jimerson, S. R., Skokut, M., Cardenas, S., Malone, H., \& Stewart, K. (2008). Where in the world is school psychology?: Examining evidence of school psychology around the globe. School Psychology International V. 29(2), 131-144. doi:10.1177/0143034308090056

Jimerson, S. R., Stewart, K., Skokut, M., Cardenas, S., \& Malone, H. (2009). How many school psychologists are there in each country of the world? International estimates of school psychologists and school psychologist-to-student ratios. School Psychology International, 30(6), 555-567. doi:10.1177/0143034309107077

Kim, K., \& Seo, E. (2018). The relationship between teacher efficacy and students' academic achievement: A meta-analysis. Social Behavior and Personality, 46(4), 529-540. doi:10.2224/sbp.6554

Koehler, S. E., \& Mata, L. (2019). Escala de Autoeficácia para Psicólogos em Contexto Escolar: Processo de construção. Em V. Monteiro, L. Mata, M. Martins, J. Morgado, J. Silva, A. C. Silva, \& M. Gomes (Eds.), Educar hoje: Diálogos entre psicologia, educação e currículo (pp. 261-275). Lisboa - Portugal: ISPA - Instituto Universitário.

Lopes, J., \& Almeida, L. S. (2015). Questões e modelos de avaliação e intervenção em Psicologia Escolar: O caso da Europa e América do Norte. Estudos de Psicologia (Campinas), 32(1). doi:10.1590/0103-166X2015000100007

Marinho-Araujo, C. M., \& Almeida, S. F. C. (2014). Psicologia Escolar: Construção e Consolidação da Identidade Profissional. (4º ed.). Campinas: Alínea.

Mata, L., Santos, N., \& Koehler, S. E. (2018). Perceção de Autoeficácia de Psicólogos a trabalhar em contexto escolar. Paper presented at the $4^{\circ}$ Congresso da Ordem dos Psicólogos Portugueses, Altice Forum Braga, Braga-PT.

Matteucci, M. C., \& Coyne, J. (2017). School psychology in Italy: Current status and challenges for future development. ISPA World Go Round 4, volume 45.

Moreira, T. C., Ambiel, R. A. M., \& Nunes, M. F. O. (2018). Escala de Fontes de Autoeficácia para Escolha Profissional: Construção e estudos psicométricos iniciais. Trends in Psychology, 26(1), 47-60. doi:10.9788/tp2018.1-03pt

Nunes, M. F. O. (2008). Funcionamento e desenvolvimento das crenças de autoeficácia: Uma revisão. Revista Brasileira de Orientação Profissional, V.9, 29-42

Nunes, M. F. O., \& Noronha, A. P. P. (2008). Escala de autoeficácia para atividades ocupacionais: Construção e estudos exploratórios. Paidéia, 18, 111-124. doi:1677-0471

Oakland, T. D., \& Jimerson, S. R. (2007). School psychology internationally: A retrospective view and influential conditions. Em S. R. Jimerson, T. D. Okland, \& P. T. E. Farrell (Eds.), The Handbook of international school psychology (pp. 453-463). London: Sage Publications.

Pacheco, E. O. (2011). Institutos federais uma revolução na educação profissional e tecnológica. Brasília: Editora Moderna. 
Pajares, F., \& Olaz, F. (2008). Teoria social cognitiva e autoeficácia: Uma visão geral. Em A. Bandura, R. G. Azzi, S. Polydoro, \& e. col. (Eds.), Teoria Social Cognitiva: conceitos básicos (pp. 97-114). Porto Alegre: Artmed.

Pedrazza, M., Trifiletti, E., Berlanda, S., \& Bernardo, G. A. (2013). Self-efficacy in social work: Development and initial validation of the Self-Efficacy Scale for Social Workers. Social Science, vol. 2, 191-207. doi:10.3390/socsci2030191

Peretta, A. A. C. S., Silva, S. M. C., Souza, C. S., Olivira, J. O., Barbosa, F. M., Sousa, L. R., \& Rezende, P. C. (2014). O caminho se faz ao caminhar: Atuações em Psicologia Escolar. Revista Quadrimestral da Associação Brasileira de Psicologia Escolar e Educacional, 18(2), $293-301$. doi:10.1590/2175-3539/2014/0182747

Petroni, A. P., \& Souza, V. L. T. (2014). Psicólogo escolar e equipe gestora: Tensões e contradições de uma parceria. Psicologia: Ciência e Profissão, 34(1), 444-459. doi: 10.1590/1982-3703000372013

Polydoro, S.A.J., \& Guerreiro-Casanova, D. C. (2010). Escala de auto-eficácia na formação superior: Construção e estudo de validação. Avaliação Psicológica, 9, 267-278. Recuperado de http://pepsic.bvsalud.org/scielo.php?script=sci_arttext\&pid=S1677-04712010000200011

Poulou, M., Reddy, L., \& Dudek, C. (2018). Relation of teacher self-efficacy and classroom practices: A preliminary investigation. School Psychology International, 40(1), 25-48. doi:10.1177/0143034318798045

Regmi, P. R., Waithaka, E., Paudyal, A., Simkhada P, \& van Teijlingen, E. (2016). Guide to the design and application of online questionnaire surveys. Nepal J Epidemiol, 6(4), 640-644. doi:10.3126/nje.v6i4.17258

Reschly, A., \& Christenson, S. L. (2019). Family engagement, partnerships, and school support personnel connections for enhancing student outcomes. Em S. B. Sheldon \& T. A. Turner (Eds.), The Wiley Handbook of Family, School, and Community Relationships in Education.

Schaufeli, W. B., Salanova, M., González-Romá, V., \& Bakker, A. B. (2002). The measurement of engagement and burnout: A confirmatory factor analytic approach. Journal of Happiness Studie, 3, 71-92. doi:10.1023/A:1015630930326

Schreiber, J., Nora, A., Stage, F., Barlow, L., \& King, J. (2006). Confirmatory factor analyses and structural equations modeling: An introduction and review. Journal of Educational Research, 99(6). doi:279291866

Shoji, K., Cieslak, R., Smoktunowicz, E., Rogala, A., Benight, C., \& Luszczynska, A. (2016). Associations between job burnout and selfefficacy: A meta-analysis. Anxiety, Stress and Coping, 29(4), 367-386. doi:10.1080/10615806.2015.1058369

Skaalvik, E., \& Skaalvik, S. (2014). Teacher stress and teacher self-efficacy as predictors of engagement, emotional exhaustion, and motivation to leave the teaching profession. Creative Education, 7, 1785-1799. doi:10.4236/ce.2016.713182

Street, K. E. S., Malmberg, L., \& Stylianides, G. J. (2017). Level, strength, and facet-specific self-efficacy in mathematics test performance. ZDM Mathematics Education, 49, 379-395. doi: 10.1007/s11858-017-0833-0

Talsma, K., Schüz, B., Schwarzer, R., \& Norris, K. (2018). I believe, therefore I achieve (and vice versa): A meta-analytic cross-lagged panel analysis of self-efficacy and academic performance. Learning and Individual Differences, 61, 136-150. doi:10.1016/j.lindif.2017.11.015

Van Overschelde, J. P., \& Lasser, J. (2019). School psychology workforce trends: Recommendations for graduate preparation and policy. Contemporary School Psychology, 1-9. doi:10.1007/s40688-019-00237-0

Vega, D., Lasser, J., \& Fernandez, S. (2017). School psychologists' family-school partnering experiences with Latinos. Psychology in the Schools, 54, 169-182. doi:https://doi.org/10.1002/pits.21988

Walcott, C., \& Hyson, D. (2018). Results from NASP 2015 emmbership survey, part one: Demographics and employment conditions. Bethesda, MD: National Association of School Psychologists.

Watt, H. M. G., Ehrich, J., Stewart, S. E., Snell, T., Bucich, M., Jacobs, N., ... English, D. (2019). Development of the Psychologist and Counsellor Self-Efficacy Scale. Higher Education, Skills and Work-Based Learning. doi:10.1108/HESWBL-07-2018-0069

Zee, M., \& Koomen, H. (2016). Teacher self-efficacy and its effects on classroom processes, student academic adjustment, and teacher wellbeing: A synthesis of 40 years of research. Review of Educational Research, 86(4), 981-1015. doi:10.3102/0034654315626801

\section{Sobre as autoras}

Solange Ester Koehler é psicóloga do Instituto Federal de Educação, Ciências e Tecnologia Farroupilha, doutoranda em Psicologia da Educação pelo ISPA - Instituto Universitário de Ciências Psicológicas, Sociais e da Vida - Lisboa - Portugal.

Lourdes Mata graduada em Psicologia Educacional pelo ISPA e com o doutoramento em Estudos da Criança pela Universidade do Minho. É Professora Auxiliar no ISPA - Instituto Universitário de Ciências Psicológicas, Sociais e da Vida - Portugal. É também membro do Centro de Investigação em Educação (CIE-ISPA, FCT-UID/CED/04853/2016). 